Dicenda. Estudios de lengua y literatura españolas

ISSN-e: $1988-2556$

http://dx.doi.org/10.5209/DICE.62153

\title{
Algunas notas sobre la gramática de las subordinadas adverbiales a propósito de Pavón Lucero (2016) $)^{1,2}$
}

\author{
Pablo Tagarro Melón (Universidad de Salamanca/Universidade de Vigo) \\ Nerea Suárez González (Universitat Pompeu Fabra)
}

El concepto de 'oración subordinada adverbial' (o 'subordinada circunstancial') ha resultado ser, sin lugar a dudas, uno de los temas más controvertidos y más complejos de la gramática del español de los últimos tiempos, de manera particular por la cantidad de estructuras que se engloban en su haber. Parece ser que la clasificación tripartita de la oración compleja en subordinadas sustantivas, adjetivas y adverbiales/circunstanciales no es suficientemente efectiva desde un punto de vista sintáctico ${ }^{3}$. La clasificación que se ofrece de las mismas, por un lado, parece estar motivada por criterios puramente semánticos (adverbiales de tiempo, lugar, causa, finalidad.... $)^{4} \mathrm{y}$, por otro lado, no todas las correspondencias (criterio de conmutación) entre subordinada adverbial - sintagma adverbial se manifiestan de manera unívoca -uno de los motivos principales por el que se estableció la diferencia entre adverbiales propias y adverbiales impropias. ${ }^{5}$ Podemos encontrar, sin embargo, diferentes aportaciones que ya han promovido diferentes análisis y soluciones al complejo fenómeno de la subordinación adverbial (vid. Bosque (1989: 210-217), Narbona (1989, 1990), Brucart y Gallego (2009), Gallego (2011), Pavón (1999, 2003, 2010, 2012), entre otros muchos $^{6}$ ). En definitiva, podemos destacar que

$1 \quad$ M. ${ }^{a}$ Victoria Pavón Lucero (ed.), Las relaciones interoracionales en español. Categorias sintácticas y subordinación adverbial, Berlín y Nueva York, Mouton de Gruyter (Beihefte zur Zeitschrift für romanische Philologie 398), 2016, 345 pp.

2 Nos gustaría agradecer la atenta lectura de una primera versión de esta recensión a los profesores (por orden alfabético) Julio Borrego Nieto (USAL) y Violeta Demonte Barreto (UAM). Otros comentarios y percepciones de esta obra se pueden consultar, por ejemplo, en la reseña de Hernández Ortega (2018). Pablo Tagarro Melón agradece la ayuda proporcionada por el Ministerio de Economía, Industria y Competitividad (ahora Ministerio de Ciencia, Innovación y Universidades) BES-2017-079864 dentro del proyecto FFI2016-77018-P del grupo de investigación Language Variation and Textual Categorisation (LVTC) de la Universidade de Vigo.

3 Vid. Zamorano Aguilar (2004) para un repaso gramaticográfico sobre el análisis de las subordinadas adverbiales en algunas gramáticas del español desde J. A. Pérez Rioja hasta E. Alarcos Llorach. Una relación de las obras con información más exhaustiva puede consultarse en Zamorano Aguilar (2004: 322-323). También pueden resultar de interés los comentarios realizados en las secciones sobre subordinación de Garrido Vílchez (2010), para las gramáticas de la Real Academia Española.

4 Pavón (2003) denominó a las conjunciones introductorias de subordinadas adverbiales como conjunciones subordinantes léxicas (CSLs) precisamente por su carácter semántico inherente. Para más información consúltese, sobre todo, Pavón (2003: 201-235).

5 Tal distinción parece atribuirse a Narbona (1989), tal y como pone de manifiesto Pérez Jiménez (2016).

6 La revisión de las diferentes posturas ocuparía un espacio del que no disponemos aquí. Dirigimos al lector interesado a dichas publicaciones, donde podrá encontrar otras muchas referencias existentes en la bibliografía 
"[d]esde un punto de vista sintáctico, las diferentes clases de oraciones subordinadas adverbiales [...] no se corresponden con una estructura unitaria" (Pavón 2012: 22; cursiva nuestra).

Por ejemplo, la última gramática de la RAE y ASALE, la Nueva gramática de la lengua española (NGRAE) (2009), no sostiene una visión estrictamente tradicional $\mathbf{l}^{7}$. Las oraciones introducidas por las partículas donde, como y cuan$d o$ se analizan como adverbios relativos en construcciones de relativo libre (NGRAE $\S 1.13 \mathrm{~s}, 22.7,22.8,22.9,22.10$ y 22.11$)$. Una de las pruebas aducidas es que, a diferencia de las conjunciones (subordinantes), estas partículas poseen una función sintáctica, complemento circunstancial en la mayoría de los $\operatorname{casos}^{8}$. Esto resulta especialmente cauteloso puesto que en otras lenguas, como el inglés, la polémica también se materializa por parte de algunos gramáticos ${ }^{9}$. La clasificación de una de las gramáticas más relevantes del inglés contemporáneo, A Comprehensive Grammar of the English Language (CGEL) (Quirk et al. 1985), sigue un criterio de corte tradicionalista ${ }^{10}$. Otras gramáticas clave posteriores representan análisis mucho más innovadores. Por ejemplo, Huddleston y Pullum et al. (2002: 1011-1017) ofrecen una serie de argumentos de índole sintáctica ${ }^{11}$ para finalmente determinar que las tradicionalmente consideradas como oraciones adverbiales son en realidad sintagmas preposicionales cuyo núcleo sería lo que en la tradición ha venido denominándose como con-

actual. Hemos tratado de remitir al lector a trabajos de carácter descriptivo, sin una preferencia en particular por alguna teoría lingüística, como es el caso, por poner un ejemplo, de Conti Jiménez (2012) que analiza el fenómeno de la subordinación adverbial en español desde la corriente funcionalista de la Gramática del Papel y la Referencia (RRG).

$7 \quad$ Vid. $\S 1.13 \mathrm{q}$ y ss.

$8 \quad$ Vid. NGRAE $(\S \S 22.7 \mathrm{a}, 22.7 \mathrm{~b}, 22.7 \mathrm{c}, 22.7 \mathrm{~d}$ y $22.7 \mathrm{e})$, para un visionado sobre las diferentes pruebas sintácticas.

9 Para una visión desde la tipología lingüística sobre el fenómeno de la subordinación, sin un énfasis en particular por las construcciones adverbiales, vid. Cristofaro (2005), y para una misma óptica tipológica con especial atención en la subordinación adverbial, vid. Thompson y Longrace (1985), Kortmann (1997, 2001), Hengeveld (1998), Diessel (2001, 2013), Dryer (2005), Thompson, Longrace y Hwang (2007), Lin (2015) o Conti Jiménez (2016: 195-204), entre otros. Es interesante resaltar que "[e]l hecho de que las cláusulas externas al predicado se hayan incorporado tarde a los estudios de sintaxis tanto en las lenguas de mayor tradición gramatical como en las lenguas de tradición más reciente explica en parte por qué no hay una tipología interlingüística de estas construcciones" (Conti Jiménez 2016: 195).

10 La clasificación que se ofrece en dicha gramática de las oraciones adverbiales es la siguiente: clauses of time, clauses of contingency, clauses of place, clauses of condition, clauses of concession, clauses of contrast, clauses of exception, clauses of purpose, reason clauses, clauses of result, clauses of proportion, clauses of preference, clauses of similarity and comparison y comment clauses, vid. Quirk et al. (1985: 1077-1118). En lo que respecta a su estatus sintáctico, se siguen, en la mayoría de los casos, las correspondencias empleadas para el análisis de los complementos adverbiales (ing. adverbials) (adjunct, subjunct, disjunct y conjunct), siendo las más habituales las de adjunct y disjunct, vid. Quirk et al. (1985: 1068-1077). Según Denison, dos son sus funciones sintácticas fundamentales en inglés: "[a]dverbial clauses can function either as arguments of a higher verb or as (optional) adjuncts" (1998: 293). Del mismo modo, pueden resultar de interés los comentarios realizados en Häcker (1999: 21-48), a pesar de ser ya antiguos, o las ideas expuestas en Verstraete (2007) desde la interfaz entre sintaxis, semántica y pragmática.

11 Para una lectura sobre las pruebas, vid. Huddleston y Pullum et al. (2002: 1011-1017). Una contrarréplica a la postura empleada por The Cambridge Grammar of the English Language (CaGEL) (2002) se puede consultar en Leech (2004: 131-134): "the attempt to elevate the preposition to a major word category is unconvincing, and seems to hard back historically to Chomsky's feature theory of word categories [...], now largely discarded" (Leech 2004: 134). 
junción subordinante, más una oración de contenido (ing. content clause) ${ }^{12}$ que funciona como término dentro de ese $\mathrm{SP}^{13,14}$. Resultaría difícil aplicar este último análisis al español ya que "una preposición no encabezaría nunca una oración con verbo dotado de flexión, pues se necesita siempre la presencia de la conjunción que" (Rodríguez Ramalle 2015: 26) ${ }^{15}$. La intervención de la partícula que nos permite, por lo tanto, un análisis alternativo de las tradicionales locuciones y conjunciones de tipo subordinante: aunque, además de que ${ }^{16}$ (adverbio $+(d e)+$ oración completiva con que), porque, a fin de que, a medida que (preposición + (nombre) + (preposición) + oración completiva con que), dado que, pese a que (elemento verbal + (preposición) + oración completiva con que), por mencionar algunos casos. No siempre se trata de una oración completiva con que, pues en algunos casos como ahora que o siempre que se considera la oración con que como una relativa (vid. pp. 34-36 del libro o Pavón (2012: 37)) y, además, otras partículas como conque o de que (< desque)

12 Para una visión sobre la distribución de las oraciones complejas en CaGEL, vid. Huddleston y Pullum et al. (2002: 949-950); y para una explicación de las content clauses en relación con la clasificación tradicional de las oraciones subordinadas, vid. Huddleston y Pullum et al. (2002: 1014-1017). El término content clause parece proceder del libro Essentials of English Grammar (1964) de Jespersen (Aarts 2006: 252).

13 En ningún caso debe caerse en la tentación de pensar que dicho análisis es pionero de la $C a G E L$, aunque probablemente sí el lugar donde se trata con más exhaustividad hasta la fecha: "[i]t should not take 250 years for observations as simple and cogent as those of Kirkby and Hunter to gain currency. Yet that is what happened. The first systematic descriptive grammar of English to adopt the lines proposed by Kirkby in 1746 [A New English Grammar] and Hunter in 1784 ['A grammatical essay in the nature, import and effect of certain conjunctions'] came more than two centuries later, in [CaGLE]" (Pullum 2015: 14). Además, podemos encontrar una serie de descripciones posteriores en Huddleston y Pullum (2004, 2006: 210-217) así como algunas sugerencias de tipo preliminar, en detrimento de los presupuestos tradicionales, een Huddleston (1984: 338-341). Sea como fuere, CaGEL parece haber tomado como fuente principal Emonds (1972), tal y como mencionan los autores en Huddleston y Pullum (2005: 293). Según Aarts, el primer autor en proponer tal análisis fue el lingüista danés: "[i]t was Jespersen who first suggested that 'before' is in fact a preposition in all three examples" (2016: 30, allí podrán encontrarse los ejemplos con before a los que se hace mención). Vid. también Aarts (2011: 74-80, 154-158 y 180-181) para una visión alternativa de corte no tradicional y basada, mutatis mutandis, en los supuestos anteriores. Según Pullum (ibid.), el origen podría situarse en el siglo XVIII. En Aarts (2006: 252) la clasificación del sistema de subordinación se organiza de la siguiente manera: oraciones de contenido (ing. content clauses), oraciones comparativas (ing. comparative clauses), oraciones de relativo (ing. relative clauses) y oraciones de adjunto (ing. adjunct clauses). Otros autores intermedios (Emonds, Jackendoff, Larson...), en su mayoría partiendo desde los preceptos del generativismo (Principios y Parámetros), presentan propuestas similares, como bien explica la editora del tomo a reseñar en el capítulo 1 (pp. 16-18) o Pavón (2003: 225-233) para un desarrollo de las propuestas. Un análisis de las adverbiales en español desde la gramática generativa se puede consultar en Pérez Jiménez (2016). Para el caso del inglés pueden resultar de interés los comentarios expuestos en Haegeman (2010), a pesar de centrarse en construcciones muy concretas, o Geis (1970), por mencionar dos de ellos. Para una panorámica de las adverbiales en inglés desde los supuestos del funcionalismo, vid. Pérez Quintero (2000a, 2000b). Para un estudio de las oraciones adverbiales en inglés americano coloquial vid. Ford (1993) y para Scots vid. Häcker (1999). Observaciones sobre diferentes aspectos de la subordinación en inglés en general se pueden encontrar en la colección de artículos editada por Seoane, Acuña Fariña y Palacios Martínez (2018) y, particularmente, Leuckert (2018) para un estudio sobre las adverbiales con since y while en ENL (English as a Native Language), ESL (English as a Second Language) y EFL (English as a Foreign Language).

14 Por razones obvias de espacio no nos hemos parado a analizar casos particulares (y peculiares), como la partícula when.

15 Especialmente interesantes resultan los comentarios realizados en el capítulo de Rosa María Espinosa (pp. 112115) o el artículo de Antonio Fábregas en el volumen a reseñar aquí.

16 Nótese que el análisis de ciertas partículas como porque o aunque se entienden, desde una perspectiva composicional, como constituidas por dos elementos: por/aun + que. El hecho de que se escriban como una sola unidad gráfica es una mera cuestión convencional de ortografía. Debemos, por consiguiente, fijarnos en su estructura profunda y no en la superficial. 
tampoco admiten un desglose (vid. Pavón 2012: 30-31) ${ }^{17}$. Por último, es interesante mencionar que existe un tipo de construcciones, las tradicionalmente catalogadas como causales, concesivas, consecutivas, condicionales y adversativas, que algunos autores han preferido denominar como oraciones bipolares por el carácter de mutua exigencia que exhiben sus dos constituyentes últimos: oración subordinada y oración principal (vid. García Berrio (1970), Moya Corral (1996), Narbona (1983, 2001), Rojo (1978: 103-112), entre otros). Esta formulación ha dado paso al planteamiento de un tercer (o cuarto si se considera la yuxtaposición) tipo de unión oracional: la interordinación ${ }^{18}$. Además, cabe mencionar que algunos estudios sobre las restricciones del modo verbal en las subordinadas adverbiales están accesibles en Veiga y Mosteiro Louzao (2006) o Pérez Saldanya (1999), por mencionar dos de ellos.

Tras esta breve introducción de carácter preliminar sobre la teoría sintáctica general de las adverbiales ${ }^{19}$, consideremos ahora la distribución general de los capítulos del volumen que aquí nos ocupa lugar.

El libro alberga un total de 9 capítulos elaborados por diferentes investigadores de primera línea en la materia. Del mismo modo, estas nueve contribuciones se encuentran agrupadas en dos macrosecciones diferentes: una primera sección (Parte I: Cuestiones generales y estudios diacrónicos ${ }^{20}$ ), donde se reúnen tres estudios de índole histórica y una extensa introducción a cargo de la propia editora, que sirve para poner al lector en contacto con los diferentes análisis y controversias por los que ha pasado el concepto ¿sintáctico? de subordinación adverbial. Una segunda sección (Parte II: Estudios sincrónicos), donde se albergan cinco capítulos, se reserva para el análisis y descripción de diferentes aspectos relacionados con las adverbiales en la lengua presente. Una breve introducción (pp. 1-8) presenta el volumen y en él se declaran las intenciones de la obra, así como se esboza una breve descripción de cada uno de los capítulos.

Parte I: Cuestiones generales y estudios diacrónicos. El capítulo 1, "Relaciones entre oraciones y subordinación adverbial” (pp. 11-39) de María Victoria Pavón Lucero (Universidad Carlos III), supone una puesta en escena de los principales presupuestos metodológicos y análisis que han recibido las oraciones adverbiales en las últimas décadas. Algunas de estas propuestas ya habían sido presentadas por la autora en otro monográfico anterior, vid. Pavón (2012). Se realiza un recorrido por los diferentes aspectos que parecen caracterizar la subordinación adverbial. Se comienza con una diferenciación entre los mecanismos de combinación oracional y las subordinadas adverbiales, así como las diferentes estructuras que rigen el

17 La cuestión no es tan sencilla como parece y, dado que las restricciones de espacio no nos permiten realizar un análisis exhaustivo de todos los casos y sus pruebas de discriminación formal correspondientes, se recomienda la consulta de: Pavón (2012: 25-32) para adverbiales en sintagmas preposicionales, Pavón (2012: 33-40) para adverbiales en sintagmas adverbiales, Pavón (2012: 41-52) para construcciones introducidas por adverbios relativos, Pavón (2012: 53-59) para adverbiales en estructuras cuantificativas y Pavón (2012: 61-70) para adverbiales introducidas por conjunciones (simples y locuciones). Algunos de los análisis ya se pueden encontrar en Pavón (1999, 2003).

18 Para una argumentación sobre la existencia de cosubordinación en español vid. Conti Jiménez (2018).

19 Para un análisis más profundo sobre la sintaxis, la casuística y la estilística de los diferentes tipos de construcciones adverbiales, se remite al lector a la consulta de los capítulos concernientes en Bosque y Demonte (dirs.) (1999). Referencias bibliográficas adicionales se pueden encontrar en tales capítulos. También sería de considerable interés observar las apreciaciones que se realizan en las secciones dedicadas al tema en la NGRAE (2009).

20 El lector interesado en cuestiones de raigambre histórica puede encontrar una revisión de los diferentes constructos adverbiales en los capítulos correspondientes de la tercera parte de la Sintaxis histórica de la lengua española (2014), dirigida por Concepción Company Company. 
funcionamiento de estas últimas. También se introducen y definen los diferentes conceptos que parecen estar asociados a las construcciones adverbiales en la bibliografía, por ejemplo, la distinción entre subordinadas adverbiales del enunciado y subordinadas adverbiales de la enunciación ${ }^{21}$. Por último, se pasa revista a los diferentes elementos que sirven para introducir dichas construcciones, aspecto que, como ya hemos revisado, resulta ser muy heterogéneo. Este capítulo supone, a nuestro parecer, uno de los mayores aciertos en lo que a la configuración general del volumen se refiere.

El capítulo 2, "Los adverbios y la estructura informativa en el margen preverbal de las oraciones subordinadas en español antiguo" (pp. 41-99) de Cristina Matute (Saint Louis University) y Teresa María Rodríguez Ramalle (Universidad Complutense de Madrid), estudia el funcionamiento y posición de los adverbios en la periferia izquierda/margen preverbal de las oraciones subordinadas del español medieval ${ }^{22}$. El estudio parte de un corpus de textos fechados entre los ss. XIII-XV y analizado manualmente. Las autoras confirman dos hipótesis que plantean al comienzo del capítulo: por un lado, los adverbios se relacionan con las categorías funcionales que se identifican en la periferia izquierda y su establecida colocación jerárquica. Por otro lado, la aparición de esas categorías funcionales depende de los rasgos particulares de los adverbios y del sintagma fuerza (SFuerza) (i.e. categoría que guarda relación con la información relacionada con la fuerza ilocutiva de la oración).

El capítulo 3, "Los cambios en las fronteras entre relativos, interrogativos y conjunciones" (pp. 101-122) de Rosa María Espinosa Elorza (Universidad de Valladolid), parte de la diferencia entre los conceptos de 'hipotaxis' (i.e. "se caracteriza por interdependencia de un núcleo y un margen, cada uno con su curva de entonación” (p. 102)) y 'subordinación' (i.e. "completa dependencia, es decir, por la incrustación del margen en el núcleo, lo que implica pérdida de estatus oracional (desententialization)" (p. 102)) para esbozar la evolución de diferentes partículas en subordinadas adverbiales del español actual. Establece que la subordinación y la hipotaxis sindética convergieron, en sus orígenes, en construcciones de hipotaxis correlativa con adverbios relativos (explicativos y especificativos). En el paso de la hipotaxis correlativa a la hipotaxis sindética se dio lugar a conjunciones hipotácticas de tipo concesivo, condicional y causal (reanálisis de los adverbios relativos explicativos como conjunciones hipotácticas). En el paso de la hipotaxis correlativa a la subordinación se analizan tres tipos de partículas/resultados: (i) adverbios relativos especificativos (donde, como, cuando), (ii) adverbios interrogativos (dónde, cómo, cuándo), y (iii) la conjunción completiva como. También se comenta el paso de ciertos adverbios relativos especificativos (grupo (i)) a preposiciones. Extremadamente esclarecedor resulta ser el Esquema 3 (p. 119), pues permite seguir el hilo argumental del capítulo con relativa facilidad. Finalmente, conviene destacar cómo la propuesta establecida por la autora al principio del capítulo (no hay que partir de un supuesto sistema relativo-interrogativo para la formación de conjunciones subordinantes puesto que los relativos, por una parte,

21 Esta distinción, primeramente aplicada al estudio de las oraciones causales, parece remontar sus orígenes a la Gramática de la lengua castellana, destinada al uso de los americanos (1847) de Andrés Bello, aunque sea de forma embrionaria. Vid. Iglesias Bango (1997) para mayor detalle sobre la génesis y desarrollo de la cuestión. Los términos del enunciado y de la enunciación parecen proceder de Marcos Marín (1979).

22 En esta línea puede resultar de interés el estudio de Haegeman (2012), entre otros. 
y los interrogativos, por otra, han llevado destinos evolutivos diferentes) parece confirmarse.

En el capítulo 4, "Cuando la gramaticalización se detiene: el caso de durante que y mediante que" (pp. 123-157) de Cristina Sánchez López (Universidad Complutense de Madrid), se examina el porqué de que ciertas preposiciones, mediante y durante (preposiciones impropias o imperfectas), procedentes de participios de presente latinos, no hayan heredado las mismas restricciones combinatorias que otros elementos de la categoría preposición, a saber: tomar oraciones como términos (oraciones de infinitivo y oraciones completivas/sustantivas introducidas por que). Dentro de su argumentación, la autora parte de supuestos históricos para establecer que dicha restricción sí era permitida por las preposiciones durante y mediante en estadios precedentes de la lengua. De hecho, esta restricción es posible encontrarla en ciertas variedades del español americano, pero no en el peninsular, con la preposición mediante. La explicación general que la autora aduce para este comportamiento sintáctico es que otras partículas (cuando, mientras, puesto que, ya que...) ya habían adquirido dichas pautas -el proceso de gramaticalización se detuvo.

Parte II: Estudios sincrónicos. El capítulo 5, "Aspectos formales e interpretativos de la subordinación adverbial” (pp. 161-199), de José María Brucart (Universitat Autònoma de Barcelona) y Ángel J. Gallego (Universitat Autònoma de Barcelona), supone un estudio que, partiendo de las bases ya propugnadas en Brucart y Gallego (2009), concluye estableciendo que las construcciones adverbiales no se ajustan a ningún patrón sintáctico independiente, como bien ha parecido defender la tradición. Según estos dos autores, los patrones de las construcciones adverbiales se adjuntarían bien al grupo de las oraciones completivas/sustantivas, en su versión por defecto o enfática, (e.g. Lo hice para verte; Iré, por más que no quieran), bien al de las relativas (e.g. Nos trata como quiere). De hecho, los autores afirman que tan solo "hay dos mecanismos básicos de subordinación, ensamble y movimiento" (p. 194), con las siguientes correspondencias: completiva por defecto - mecanismo de ensamble externo, completiva enfática - mecanismo de ensamble externo o interno (movimiento), y relativas - mecanismo de ensamble interno (movimiento). Siendo los últimos dos mecanismos resultado de una transformación de desplazamiento.

El capítulo 6, "Cómo, cuándo y dónde donde, como y cuando se emplean como preposiciones” (pp. 201-231) de Antonio Fábregas (Universitetet i Tromsø), estudia las propiedades gramaticales de lo que el autor considera, como otros muchos, adverbios relativos: mientras, como, donde y cuando. Para ello parte del basamento teórico de la nanosintaxis ${ }^{23}$. El autor intenta ahondar, a partir de las secciones 4 y 5 , en las diferencias entre mientras y el resto de adverbios relativos considerados. Una de las diferencias es que mientras no puede materializar un antecedente explícito

23 Se trata de una teoría sintáctica que "tiene como base la idea de que los exponentes morfofonológicos se relacionan directamente con la estructura sintáctica, sin intermediación de niveles morfológicos o de adaptación - contra la Morfología Discreta” (p. 209). Además de esta idea anterior de que no hay niveles de análisis morfológicos, la nanosintaxis se fundamenta en dos principios generales: (1) "La primera es la llamada materialización de sintagma (Phrasal spell out): las entradas léxicas relacionan estructuras sintagmáticas con exponentes morfofonológicos (y su semántica conceptual)" (p. 209), y (2) "El segundo principio que define la nanosintaxis es el llamado Principio de Lexicalización Exhaustiva: frente a la Morfología Discreta y otras teorías donde el léxico puede dejar de lexicalizar un rasgo sintáctico [...] en nanosintaxis todos los rasgos sintácticos deben estar identificados por una pieza léxica" (p. 210). 
y tampoco analizarse como preposición. La solución que se propone, a partir de la teoría sintáctica utilizada, es que mientras no contiene un patrón preposicional en su entrada léxica, dado que todos los rasgos sintácticos tienen que estar determinados por un ítem léxico en nanosintaxis.

En el capítulo 7, "Rasgos gramaticales de adverbios y pronombres relativos en construcciones existenciales" (pp. 233-280) de Edita Gutiérrez (Universidad de Castilla-La Mancha) y Pilar Pérez Ocón (Universidad de Castilla La-Mancha), se estudian un tipo de construcciones a las que las autoras se refieren como 'construcciones de relativo libres de interpretación indefinida' (RLI) (e.g. No tiene a quién hablar). El título del capítulo no es en absoluto azaroso, pues tan solo se estudian las construcciones RLI complemento de predicados existenciales (i.e. con los verbos haber y tener), dejando de lado una serie de predicados intensionales (i.e. buscar, encontrar...) donde también pueden aparecer. Este tipo de construcciones son de análisis cuestionable, dado que se encuentran próximas a las interrogativas indirectas y a las relativas libres (RL). Las autoras finalmente analizan dichas construcciones como "proyecciones nominales cuantificativas encabezadas por un cuantificador vacío, semejante a nadie o alguno, de interpretación indefinida e inespecífica y que es un término de polaridad modal" (p. 277) - las RLI son un tipo de RL.

El capítulo 8, "De la periferia oracional a la estructura interna: las condicionales argumentales" (pp. 281-302) de Hilda Albano (Universidad de Buenos Aires) y Mabel Giammatteo (Universidad de Buenos Aires), evalúa un tipo de oraciones que se encuentran a caballo entre las condicionales y las sustantivas: ¿Te importa si uso tu impresora? Se trata, a diferencia de otras construcciones condicionales, de oraciones exigidas por un verbo ('verbos de afección psicológica') y de ahí la denominación de 'condicionales argumentales'. A lo largo del capítulo se presentan las características sintácticas y semánticas, aunque también apuntes de índole pragmática y diacrónica, para poder dilucidar finalmente que se trata de oraciones funcionalmente similares a las sustantivas con que en subjuntivo $(\sim$ iTe importa que use la impresora?). Ese uso del subjuntivo parece estar motivado por el carácter eventual propio de las condicionales.

En el capítulo 9, "Las subordinadas temporales comparativas" (pp. 303-345) de Lura Brugè (Università 'Ca' Foscari' Venezia) y Avel·lina Suñer (Universitat de Girona), se describe el funcionamiento gramatical de las 'temporales comparativas' introducidas por los adverbios antes (eje temporal de anterioridad) y después (eje temporal de posterioridad). Las autoras del capítulo esgrimen que, a diferencia de otras partículas introductoras de subordinadas temporales, estás partículas conllevan rasgos computacionales de adición, en el caso de después, y de sustracción, en el caso de antes, similares al de los cuantificadores comparativos más (aditivo) y menos (sustractivo) -comparativas de desigualdad. Dichos constructos se definen, pues, como: "las subordinadas introducidas por antes y después pueden ordenar temporalmente los dos eventos que relacionan restando (antes) o sumando (después) un intervalo a un punto o segmento temporal tomado como referencia por ambos interlocutores" (p. 304). En conclusión, se analizan las propiedades formales de las construcciones introducidas por estos adverbios.

En definitiva, este monográfico viene a cumplir, a nuestro parecer, dos objetivos principales: (1) por un lado, presenta una excelente aportación sobre la panorámica actual de la sintaxis oracional relacionada con las tradicionalmente catalogadas 
como subordinadas adverbiales, presentando importantes avances dentro de este campo. (2) Por otro lado, viene a sellar una controvertida laguna de conocimiento que ya había suscitado un interés considerable por la bibliografía actual de la gramática del español. Quizá el libro podría haberse beneficiado de un índice con los conceptos y términos esenciales de cada uno de los capítulos. Esto habría permitido al investigador especializado la búsqueda de temas en particular de una forma más efectiva y rápida. De otro modo, resulta muy positivo observar cómo cada una de las contribuciones anexa una relación de las diferentes fuentes que ha/n utilizado el/los autor/es; es común encontrar hoy en día monográficos editados que ofrecen una sola nómina de fuentes al final. Sea como fuere, nos encontramos ante una novedad bibliográfica que, a partir de ahora, se convertirá en referencia obligada para todo investigador riguroso del ars grammatica de la subordinación adverbial del español.

\section{Obras citadas}

Aarts, Bas, “Otto Jespersen 1860-1943”, Babel. The Language Magazine, 14 (2016), pp. 29-31.

—, "Subordination", Enclyclopedia of Language \& Linguistics, 14 vols. (ed.) Keith Brown, Oxford, Elsevier, $2006^{2}$ [1993], pp. 248-254.

—: Oxford Modern English Grammar, Oxford, Oxford UP, 2011.

Bello, Andrés: Gramática de la lengua castellana, destinada al uso de los americanos, 2 vols., Madrid, Arco/Libros, 1988. Estudio y edición de Ramón Trujillo y notas de Rufino José Cuervo. Esta obra se publicó por primera vez en 1847 en Santiago de Chile.

Bosque, Ignacio, Las categorías gramaticales, Madrid, Síntesis, 1989. Existe una segunda edición de este libro publicada en 2015, en la misma editorial (Serie Mayor), bajo el título de Las categorías gramaticales. Relaciones y diferencias.

Bosque, Ignacio y Violeta Demonte (dirs.): Gramática descriptiva de la lengua española, 3 vols., Madrid, Espasa (Colección Nebrija y Bello), 1999.

Brucart, José María y Ángel J. Gallego, “L’estudi formal de la subordinació i l'estatus de les subordinades adverbials”, Llengua y Literatura, 20 (2009), pp. 139-191.

Company Company, Concepción (dir.), Sintaxis histórica de la lengua española. Tercera parte: Preposiciones, adverbios y conjunciones. Relaciones interoracionales, México, Fondo de Cultura Económica/Universidad Nacional Autónoma de México, 2014.

Conti Jiménez, Carmen: “Disjunctive Clauses with o. o 'either. or' in Spanish and Clausal Cosubordination", Folia Linguistica. Acta Societatis Linguisticae Europaeae, 52.1 (2018), pp. 75-106.

— , "Subordinación periférica y subordinación dependiente: clasificación estructural de la subordinación adverbial en español”, en El funcionalismo en la teoría lingüística: la Gramática del Papel y la Referencia. Introducción, avances y aplicaciones, (eds.) Ricardo Mairal, Lilián Guerrero y Carlos González Vergara, Madrid, Akal, 2012, pp. 269-286.

—, Fundamentos de sintaxis tipológica, Madrid, Síntesis (Claves de la Lingüística), 2016.

Cristofaro, Sonia, Subordination, Oxford, Oxford UP (Oxford Studies in Typology and Linguistic Theory), 2005.

Denison, David, "Syntax", The Cambridge History of the English Language. Volume IV: 1776-1997, (ed.) Suzanne Romaine, Cambridge, Cambridge UP, 1998, pp. 92-329. 
Diessel, Holger: “Adverbial Subordination”, Bloomsbury Companion to Syntax, (eds.) Silvia Luraghi y Claudia Parodi, Londres, Continuum, 2013, pp. 341-354.

- , "Competing Motivations for the Ordering of Main and Adverbial Clauses", Linguistics, 43 (2005), pp. 449-470.

- "The Ordering Distribution of Main and Adverbial Clauses: A Typological Study", Language, 77 (2001), pp. 345-365.

Dryer, Matthew S.: "Order of Adverbial Subordinator and Clause", The World Atlas of Language Structures Online, (eds.) Martin Haspelmath, Matthew S. Dryer, David Gil y Bernard Comrie, Leipzing, Oxford, Oxford UP, 2005, 382-283.

Emonds, Joseph E: "Evidence that Indirect Object Movement is a Structure-Preserving Rule", Foundations of Language, 8 (1972), pp. 546-561.

Ford, Cecilia E.: Grammar in Interaction: Adverbial Clauses in American English Conversations, Cambridge, Cambridge UP (Studies in Interactional Sociolinguistics), 1993.

Gallego, Ángel J., “Cuando, ¿preposición o adverbio relativo?”, 60 problemas de gramática dedicados a Ignacio Bosque, (eds.) M Victoria Escandell Vidal, Manuel Leonetti y Cristina Sánchez López, Madrid, Akal, 2011, pp. 9-17.

García Berrio, Antonio, "Bosquejo para una descripción de la frase compuesta en español”, Universidad de Murcia, 1970.

Garrido Vílchez, Gema Belén, Las Gramáticas de la Real Academia Española: teoría gramatical, sintaxis y subordinación (1854-1924), Salamanca, Universidad de Salamanca, 2010.

Geis, Michael: Adverbial Subordinate Clauses in English, tesis doctoral inédita, Massachusetts Institute of Technology (MIT), 1970.

Gutiérrez Rodríguez, Edita: "El laberinto de la terminología lingüística en las aulas”, Revista de Gramática Orientada a las Competencias, 1.1 (2018), pp. 203-235.

Häcker, Martina, Adverbial Clauses in Scots: A Semantic-Syntactic Study, Berlín y Nueva York, Mouton de Gruyter (Topics in English Linguistics), 1999.

Haegeman, Liliane: Adverbial Clauses, Main Clause Phenomena, and Composition of the Left Periphery. The Cartography of Syntactic Structures, Oxford, Oxford UP (Oxford Studies in Comparative Syntax), 2012.

—, "The Internal Syntax of Adverbial Clauses", Lingua, 120 (2010), pp. 628-648.

Hengeveld, Kees: "Adverbial Clauses in the Languages of Europe", Adverbial Constructions in the Languages of Europe, (ed.) Johan Van der Auwera, Berlín y Nueva York, Mouton de Gruyter, 1998, pp. 335-419.

Hernández Ortega, Juan: "Reseña de Las relaciones interoracionales en español: Categorías sintácticas y subordinación adverbial (2016), editado por M. ${ }^{\text {a }}$ Victoria Pavón Lucero", Infoling, 7/28 (2018), pp. 1-7.

Huddleston, Rodney D., Introduction to the Grammar of English, Cambridge, Cambridge UP, 1984.

Huddleston, Rodney D. y Geoffrey K. Pullum: A Student's Introduction to English Grammar, Cambridge, Cambridge UP, 2005 (segunda edición en preparación).

Huddleston, Rodney D. y Geoffrey K. Pullum et al., The Cambridge Grammar of the English Language, Cambridge, Cambridge UP, 2002.

Huddleston, Rodney D. y Geoffrey K. Pullum, "Coordination and Subordination", The Handbook of English Linguistics, (eds.) Bas Aarts y April McMahon, Oxford, Wiley-Blackwell, 2006, pp. 198-219.

Huddleston, Rodney D. y Geoffrey K. Pullum, "The Classification of Finite Subordinate Clauses", An International Master of Syntax and Semantics: Papers presented 
to Aimo Seppänen on the occasion of his 75th birthday, (eds.) G. Bergh, J. Herriman y M. Mobärg, Gotemburgo, Acta Universitatis Gothoburgensis, 2004, pp. 103116.

Iglesias Bango, Manuel, "La oposición enunciado/enunciación y las llamadas adverbiales impropias en español”, Moenia, 3 (1997), pp. 237-269.

Kortmann, Bernd: “Adverbial Clauses", International Encyclopedia of the Social \& Behavioral Sciences, 26 vols., (eds.) Neil J. Smelser y Paul B. Baltes, Oxford, Elsevier, 2001, pp. 162-167.

-, Adverbial Subordination: A Typology and History of Adverbial Subordinators Based on European Languages, Berlín y Nueva York, Mouton de Gruyter (Empirical Approaches to Language Typology), 1996.

Leech, Geoffrey, “A New Gray's Anatomy of English Grammar”, English Language and Linguistics, 8/1 (2004), pp. 121-147.

Leuckert, Sven: “Adverbial Subordination across Variety Types: A Synchronic Analysis of the Syntax and Semantics of Since- and While-Clauses in ENL, ESL, and EFL", Subordination in English: Synchronic and Diachronic Perspectives, (eds.) Elena Seoane, Juan Carlos Acuña Fariña e Ignacio Palacios Martínez, Berlín y Nueva York, Mouton de Gruyter, 2018, pp. 235-262.

Lin, Jingxia: “Adverbial Clauses", International Encyclopedia of the Social \& Behavioral Sciences, 26 vols., $2^{\mathrm{a}}$ ed., (ed.) James D. Wright, Oxford, Elsevier, 2015, pp. 185-188.

Marcos Marín, Francisco A., "A propósito de las oraciones causales. Observaciones críticas”, Cuadernos de Filología, 2/1 (1979), pp. 163-171.

Moya Corral, José Antonio, Los mecanismos de la interordinación: a propósito de "pero” y “aunque”, Granada, Universidad de Granada, 1996.

Narbona Jiménez, Antonio, "Otra vez sobre interordinación y subordinación”, Indagaciones sobre la lengua. Estudios de filología y lingüística españolas en memoria de Emilio Alarcos, (eds.) Elena Méndez, Josefa Mendoza y Yolanda Congosto, Sevilla, Universidad de Sevilla, 2001, pp. 119-138.

_, "Sobre las oraciones bipolares", Alfinge, 1 (1983), pp. 121-140.

—, Las subordinadas adverbiales impropias en español. Bases para su estudio, Málaga, Librería Ágora, 1989.

-, Las subordinadas adverbiales impropias del español (II). Causales y finales, comparativas y consecutivas, condicionales y concesivas, Málaga, Librería Ágora, 1990.

Pavón Lucero, M. ${ }^{a}$ Victoria, "Clases de partículas: preposición, conjunción y adverbio”, Gramática descriptiva de la lengua española, (dirs.) Ignacio Bosque y Violeta Demonte, Madrid, Espasa (Colección Nebrija y Bello), 1999, pp. 565-655.

- "Why Are There no Locative Conjunctions in Spanish?", Catalan Working Papers in Linguistics, 9 (2010), pp. 103-123.

-, Estructuras sintácticas en la subordinación adverbial, Madrid, Arco/Libros (Cuadernos de Lengua Española), 2012.

—, Sintaxis de las partículas, Madrid, Visor (Colección Gramática Española), 2003.

Pérez Jiménez, M. a Isabel: "Subordinación adverbial”, Enciclopedia de lingüística hispánica, 2 vols., (coord.) Javier Gutiérrez Rexach, Londres, Routledge, 2016, pp. 75-87.

Pérez Quintero, M. Jesús: Adverbial Subordination in English: A Functional Approach, Ámsterdam y Nueva York, Rodopi, 2000a.

- "Adverbial Subordination in Functional Grammar: State of the Art and Prospects", Revista Canaria de Estudios Ingleses, 40 (2000b), pp. 259-278. 
Pérez Saldanya, Manuel: "El modo en las subordinadas relativas y adverbiales", Gramática descriptiva de la lengua española, 3 vols., (dirs.) Ignacio Bosque y Violeta Demonte, Madrid, Espasa (Colección Nebrija y Bello), 1999, pp. 3253-3322.

Pullum, Geoffrey K., "The Unfortunate Divorce of English Grammar from English Literature", Philologist: Journal of Language, Literature and Culture, 11 (2015), pp. 9-20.

Quirk, Randolph, Sidney Greenbaum, Jan Svartvik y Geoffrey Leech, A Comprehensive Grammar of the English Language, Londres, Longman, 1985.

RAE y ASALE, Nueva gramática de la lengua española, 2 vols., Madrid, Espasa, 2009.

Rodríguez Ramalle, Teresa María, Las relaciones sintácticas, Madrid, Síntesis (Claves de la Lingüística), 2015.

Rojo, Guillermo, Cláusulas y oraciones, Anejo 14 de Verba. Anuario gallego de filología, Santiago de Compostela, Universidad de Santiago de Compostela, 1978.

Seoane, Elena, Juan Carlos Acuña-Fariña e Ignacio M. Palacios-Martínez (eds.), Subordination in English: Synchronic and Diachronic Perspectives, Berlín y Nueva York, Mouton de Gruyter (Topics in English Linguistics), 2018.

Thompson, Sandra A. y Robert E. Longacre: "Adverbial Clauses", Language Typology and Syntactic Description, 2 vols., (ed.) Timothy Shopen, Cambridge, Cambridge UP, 1985, pp. 171-234.

Thompson, Sandra A., Robert E. Longacre y Shin Ja J. Hwang: “Adverbial Clauses”, Language Typology and Syntactic Description, 2 vols., $2^{\mathrm{a}}$ ed., Cambridge, Cambridge UP, 2007, pp. 237-300.

Veiga, Alexandre y Manuel Mosteiro Louzao: El modo verbal en cláusulas condicionales, causales, consecutivas, concesivas, finales y adverbiales de lugar, tiempo y modo, Salamanca, Universidad de Salamanca, 2006.

Verstraete, Jean-Christophe, Rethinking the Coordinate-Subordinate Dichotomy: Interpersonal Grammar and the Analysis of Adverbial Clauses in English, Berlín y Nueva York, Mouton de Gruyter (Topics in English Linguistics), 2007.

Zamorano Aguilar, Alfonso, "Las "proposiciones adverbiales" en las gramáticas del español (1953-1994). Caracterización historiográfica y revisión crítica". Verba, 31 (2004), pp. 305-324. 